\title{
GI-MS46-P12 | Fxe Status: Femtosecond X-RAy Experiments For Chemical Dynamics Research At The European Xfel
}

Rodriguez Fernandez, Angel (European XFEL, Schenefeld, GER); Bressler, Christian (European XFEL, Schenefeld, GER); Gawelda, Wojciech (European XFEL, Schenefeld, GER); Galler, Andreas (European XFEL, Schenefeld, GER); Khakhulin, Dmitry (European XFEL, Schenefeld, GER); Zalden, Peter (European XFEL, Schenefeld, GER); Alves Lima, Federico (European XFEL, Schenefeld, GER); Kubicek, Katharina (European XFEL, Schenefeld 4, GER)

The instrument for Femtosecond X-ray Experiments (FXE) at the European X-ray Free Electron Laser (Eu XFEL) is a instrument desing for measuring the electronic and geometric sturcture changes on matter. Different techniques are available at the FXE instrument for both X-ray scattering (XRD) and X-ray emission spectroscopy (XES). XES is sensitive to electronic changes, such as oxidation and spin states, while $\mathrm{X}$-ray absorption fine structure tools deliver information about the local geometric structure around the selected absorbing atom. Combining these tools with forward scattering in one single setup allows extracting simultaneous information about the local to rather global structural changes occurring in the reacting system.

We will present some case examples, for which pico- and femtosecond x-ray experiments deliver new insight into evolving dynamic processes, including reactive high-valent iron compounds and a class of spin transition systems. This will be preceded by an introduction about the information content of $\mathrm{x}$-ray tools.

Finally, we will present the current status of this new instrument at European XFEL together with some results. 